combination of physiotherapy, medical agents and, occasionally, surgery.

An early and regular physical rehabilitation program is recommended with use of assistive devices such as ankle-foot orthoses.

As a first step, a trial of Dopamine should be considered if the diagnosis of HSP is in doubt. Furthermore some forms of HSP have shown response to Dopamine. Oral Baclofen is used as a first-line anti-spasticity drug followed by oral Gabapentin and Tizanidine. Intrathecal baclofen in selected patients has shown promising results; this should be considered early in eligible patients if oral medication is failing to achieve desired spasticity control or side effects limit oral dosing.

The evidence for the use of botulinum toxin in HSP is limited and unclear. Its benefits are greatest when combined with therapy and splinting. Diazepam can be used as an adjunct to other oral medications to improve symptom control.

Selective Dorsal Rhizotomy is not routinely offered for HSP and should only be considered for uncomplicated stable HSP when all other treatments have failed and quality of life is severely affected.

Conclusion Managing spasticity well in patients with HSP can immensely improve their quality of life. We propose a standardised referral and management pathway that summarises the approach to medical and surgical options for patients with HSP.

\section{GP117 LUMBAR PUNCTURE PERFORMANCE AND THE PAEDIATRIC PATIENT, ONE HOSPITAL'S EXPERIENCE}

${ }^{1}$ Mojahid Elbadry Ali*, ${ }^{2}$ Monica Salama, ${ }^{2}$ Michael B O'Neill. ${ }^{1}$ Cork University Maternity Hospital, Cork, Ireland; ${ }^{2}$ Mayo University Hospital, Castelbar, Ireland

\subsection{6/archdischild-2019-epa.182}

Aim To evaluate the frequency, indications, success rate and clinical impact on the performance of lumbar punctures (LP) in a paediatric population attending a general hospital.

Methods Utilising the Mayo University Hospital Microbiology database the number of CSF samples analysed was determined over a 1 year period. Failed LPs were determined using blood Polymerase Chain Reaction (PCR) testing as a surrogate marker of sepsis. Data abstracted included 1) age 2) indication for LP 3) physician performer 4) procedure quality 5) number of attempts 6) CSF analysis and 7) final diagnosis. The charts of those patients with blood PCR requested were reviewed to discern the indication for the test and an evaluation of why the LP procedure failed.

Results The number of procedures where CSF was obtained was 18 , the indications for LP were a) 5 newborns with suspected sepsis, b) 2 neonates from home with fever without focus, c) 8 infants (29-90 days) with fever without focus and irritability, d) 3 over 3 months of age; 1 with 3 afebrile seizures and 2 with fever and an unwell appearance.

? Registrars performed $94 \%$ of the procedures ? Only 1 SHO attempted a LP but had a failed attempt ? The numbers of LP with 1 attempt was 15 (83\%), 2 attempts 3 (17\%). The number of atraumatic CSF was 15 (83\%).

The final diagnoses on the CSFs were: 13 sterile cultures and 5 positive for viruses. Of the 7 children who had Blood PCR performed, 1 had LP attempted and failed after 2 trials in a child who later proved to have pneumococcal sepsis.

Conclusion LP is performed in infants rather than children. Consequently, attaining competency in LP performance will elude many Basic Specialist Trainees on their general paediatric rotations. Teaching LP performance using simulation techniques is required. Although bacterial meningitis is rare nowadays, LP is still required to evaluate cases of suspected Serious Bacterial Infection especially if no focus is found after initial assessment.

\section{GP118 FLUID PRESCRIBING FOR CHILDREN BY NON- PAEDIATRIC CLINICIANS}

Eimear McCorny . Antrim Area Hospital, UK

\subsection{6/archdischild-2019-epa.183}

Background The prescription and monitoring of fluids for paediatric patients has been very much in the forefront of paediatricians' minds within Northern Ireland over the last number of years.

Regional fluid prescription charts and guidelines have been created and compulsory learning modules, teaching and audits are common place within the paediatric setting, ensuring safe prescribing practices.

However paediatricians and paediatric trainees are not the only medical staff who may prescribe fluids for children. We aimed to examine whether doctors within other specialties who treat children are as aware or focused on the issues with paediatric fluid prescribing as we are.

Methods An anonymous questionnaire was handed out to emergency department and surgical doctors. Based on a simple, common paediatric presentation respondents were asked how they would prescribe bolus and maintenance fluids to include type of fluid, volume, rate of administration and monitoring. The answers given were assessed based on the regional paediatric fluid prescribing guidelines.

Results There were a total of 15 respondents (10 emergency department and 5 surgical) over a range of of grades (F2/ SHO/ST1/ST4/staff grade/specialty doctor).

$27 \%$ respondents would prescribe inappropriate bolus fluid. $64 \%$ respondents would prescribe inappropriate bolus volumes. $47 \%$ respondents would prescribe inappropriate maintenance fluid. $40 \%$ respondents would prescribe maintenance fluids at an inappropriate rate. 36\% respondents would check electrolytes too infrequently.

Conclusion This highlights that although much emphasis is placed on safe fluid prescribing within paediatrics the same weight may not be placed on this issue across other clinical specialties. Further teaching and training is required for all specialties involved in paediatric fluid prescribing to ensure patient safety.

\section{GP119 ANTIBIOTIC STEWARDSHIP: DETERMINING THE EXTENT OF E. COLI RESISTANCE TO CO-AMOXICLAV IN CHILDREN WITH URINARY TRACT INFECTION IN MAYO UNIVERSITY HOSPITAL}

${ }^{1}$ Monica Salama*, ${ }^{1}$ Aliya hamid, ${ }^{1}$ Claudia Stanciu, ${ }^{2}$ Burhan Ullah, ${ }^{3}$ Johannes Letshwiti. ${ }^{1}$ Mayo university Hospital, Castlebar, Ireland; ${ }^{2}$ Mullingar teaching hospital, mullingar, Ireland; ${ }^{3}$ Galway University hospital, Galway, Ireland

\subsection{6/archdischild-2019-epa.184}

Introduction A urinary tract infection is an infection in any part of the urinary system. Common symptoms in children 